\title{
Studienförderung für die akademische Nachqualifizierung von Migranten: Fehlanzeige
}

ANNA KATHARINA JACOB, MATTHIAS KNUTH

\section{Ausgangssituation}

Neueinwanderer nach Deutschland sind in zunehmendem Maße akademisch qualifiziert (siehe auch Beitrag von Galgóczi/Leschke in diesem Heft). Nach den Ergebnissen der IAB-SOEP-Migrationsstichprobe verfügten $26 \%$ der Einwanderer im Alter von 25 Jahren und älter bereits im Jahr ihrer Zuwanderung über einen akademischen Abschluss; diese Anteile sind im Zeitablauf gestiegen von $23 \%$ unter den in der zweiten Hälfte der 1990er Jahre Eingewanderten auf $36 \%$ der Neuankömmlinge ab 2005. ${ }^{1}$ Vergleicht man die Akademikerquoten der jeweiligen Bevölkerung mit eigener Migrationserfahrung mit denen der Bevölkerung ohne Migrationshintergrund (jeweils für die Altersgruppe von 25 bis 64 Jahren) für das Jahr 2013 mit dem Jahr 2005, so verringert sich bei den eingewanderten Männern der anfängliche Rückstand leicht, während die eingewanderten Frauen die einheimischen inzwischen überholt haben. ${ }^{2}$ Die aktuellen Wanderungsbewegungen bringen also in erheblichem Maße akademische Berufsabschlüsse nach Deutschland, und diese Entwicklung wird überproportional von Frauen geprägt.

Die relative Arbeitsmarktsituation der Bevölkerung mit eigener Migrationserfahrung hat sich jedoch nicht im gleichen Maße verbessert. Insgesamt leben über 900.000 Hochschulabsolventen ohne deutschen Abschluss in Deutschland - das sind nicht weniger als $10 \%$ aller Akademikerinnen und Akademiker im Alter von unter 65 Jahren. ${ }^{3}$ Doch fällt die Beschäftigungsquote unter ihnen gegenüber jenen, die über inländische Bildungszertifikate verfügen, deutlich ab. ${ }^{4}$ Sind 89,7 \% aller Akademikerinnen und Akademiker mit deutschem Schul- und Studienabschluss im Alter von 25 bis 64 Jahren erwerbstätig (und im Gegenzug lediglich
2,3\% erwerbslos), so trifft dies lediglich auf $83,6 \%$ derjenigen mit ausländischem Schul- und deutschem Studienabschluss (Erwerbslosenquote 5,3 \%) und 71,3 \% derjenigen mit ausländischem Schul- und Studienabschluss (Erwerbslosenquote 8,5 \%) zu. Dieses Problem spitzt sich geschlechtsspezifisch zu: Zwar ist die Akademikerquote unter eingewanderten Frauen höher als bei den Männern (s. o.), jedoch ist unter den Frauen mit ausländischem Abschluss die Erwerbstätigenquote niedriger und die Erwerbslosenquote höher als bei den Männern mit einem solchen Abschluss. Auch die Qualität der Beschäftigung ist schlechter: Beschäftigte Migranten aus EU-Staaten, die ihren beruflichen oder akademischen Abschluss im Ausland erworben haben, haben eine doppelt so hohe Befristungsquote wie Inländer mit Abschluss; Beschäftigte aus Drittstaaten sogar eine 2,5-mal erhöhte Befristungsquote. ${ }^{5}$ Daneben existiert das Problem der unterwertigen Beschäftigung von Migrantinnen und Migranten (vgl. auch die Beiträge von Galgóczi/Leschke und Höhne/Schulze Buschoff in diesem Heft).

\section{Anerkennung ausländischer Abschlüsse in Deutschland}

Eine gewisse Entwertung von im Ausland erworbenem Humankapital durch grenzüberschreitende Migration ist gerade bei akademischen Qualifikationen kaum zu vermeiden, da ein Teil der akademischen Fachrichtungen in erheblichem Maße kultur- und systemspezifisch geprägt ist. Andererseits rühren die Schwierigkeiten von Bildungsausländern (also Personen mit ausländischem Schul- und Studienabschluss), ihr mitgebrachtes Humankapital zu verwerten, aber auch daher, dass ihre Abschlüsse in Deutschland nicht anerkannt sind, weder im formalrechtlichen Sinne noch im Sinne
1 Brücker, H./Bartsch, S./Eisnecker, P./Kroh, M./Liebau, E./Romiti, A. et al. (2014): Die IAB-SOEP-Migrationsstichprobe: Leben, lernen, arbeiten - wie es Migranten in Deutschland geht, IAB-Kurzbericht 21/2014.

2 Statistisches Bundesamt (Hrsg.): Fachserie 1, Reihe 2.2 für 2005 und 2013. Bei dieser Gegenüberstellung bleibt die Gruppe der Personen mit Migrationshintergrund, aber ohne eigene Migrationserfahrung, außer Betracht.
3 Geis, W. (2012): Der Beitrag der Zuwanderung zur Fachkräftesicherung, in: IW-Trends 39 (2), S. 85-98.

4 Geis, W./Kemeny, F./Plünnecke, A. (2014): Rendite akademischer Nachqualifizierung für zugewanderte Hochschulabsolventen: Institut der deutschen Wirtschaft Köln, S. 14ff.

5 Bundesministerium für Bildung und Forschung (2014): Bericht zum Anerkennungsgesetz, S. 58. 
des Erkennens und der Wertschätzung durch potenzielle Arbeitgeber.

Bezüglich der formellen Anerkennung sind drei Konstellationen zu unterscheiden:

(1) Sogenannte reglementierte Berufe dürfen ohne formelle Feststellung der Gleichwertigkeit des ausländischen Abschlusses nicht ausgeübt werden - z. B. akademische medizinische Heilberufe, Medizinalfachberufe, Lehrerinnen und Lehrer an öffentlichen Schulen, Berufe des Ernährungshandwerks oder sicherheitsrelevante technische Berufe.

(2) Nicht nur für diese, sondern auch für nicht reglementierte Berufe haben das 2012 in Kraft getretene Berufsqualifikationsfeststellungsgesetz (BQFG) sowie diesem nachgebildete Anerkennungsgesetze der Länder einen verbindlichen Rahmen für die Durchführung von Anerkennungsverfahren geschaffen, an deren Ende die Feststellung der Gleichwertigkeit eines Abschlusses oder von wesentlichen Unterschieden stehen, die der Anerkennung entgegenstehen. Der Geltungsbereich dieser Anerkennungsgesetze beschränkt sich jedoch auf Berufe mit einer staatlichen Regulierungskompetenz von Bund oder Ländern.

(3) Für den Bereich der nicht reglementierten akademischen Berufe und Abschlüsse existiert dagegen kein formelles Anerkennungsverfahren. Die Lissabon-Konvention verpflichtet zwar die Vertragsstaaten, Hochschulabschlüsse wechselseitig anzuerkennen. Dieses zwischenstaatliche Recht betrifft die Hochschulen und öffentliche Arbeitgeber, bindet jedoch private Arbeitgeber nicht (die ja auch nicht verpflichtet sind, einen deutschen Bachelor-Abschluss für berufsqualifizierend zu halten). Die prinzipielle Anerkennung von Hochschulabschlüssen ohne Aussage über ihre Gleichwertigkeit ${ }^{6}$ dürfte für sie auch weniger Überzeugungskraft haben als das Verfahren nach dem BQFG, in dem die auch jeweils für die Vergabe inländischer Abschlüsse zuständige Stelle - bei den Berufen nach dem Berufsbildungsgesetz also beispielsweise die zuständige Kammer - die Gleichwertigkeit feststellt.

Für einen großen Teil der akademischen Berufe haben die Anerkennungsgesetze also keine Verbesserung hinsichtlich der Verwertungsmöglichkeiten ausländischer Abschlüsse auf dem inländischen Arbeitsmarkt gebracht - und können dieses auch gar nicht, weil diese Berufe aufgrund von Graden und Titeln ausgeübt werden, die die Hochschulen kraft ihrer Autonomie und ohne staatliche Kontrolle verleihen.

\section{Nachqualifizierung zum inländischen Abschluss als Alternative zur Anerkennung des ausländischen}

Wie die eingangs genannten Zahlen deutlich machen, konnte eine Vielzahl ausländischer Akademikerinnen und Akademiker trotz des von Vertretern der Wirtschaft angezeigten Fachkräftemangels bislang nicht adäquat in den hochqua- lifizierten deutschen Arbeitsmarkt integriert werden. Dies liegt in vielen Fällen daran, dass der ausländische Studienabschluss hierzulande formal oder faktisch nicht anerkannt wird. In vielen Fällen war der Aufbau des Studiengangs nicht Bologna-konform (bei älteren Diplomen), ${ }^{7}$ oder die Studienländer haben die Lissabonner Konvention nicht ratifiziert. Letzteres gilt für zahlreiche der Interessenten aus Ländern außerhalb Europas.

Vielfach ist der ausländische Studiengang und -abschluss bei deutschen Arbeitgebern auch einfach unbekannt und weckt Unsicherheit bezüglich der erwartbaren Qualifikationen. Da ein deutscher Studienabschluss nachweislich die Anstellungschancen am inländischen Arbeitsmarkt steigert (s. o.), erschien es als möglicher Weg, Nachqualifizierungsmaßnahmen in einem ebensolchen münden zu lassen, der sich jedoch durch Anrechnung von im Ausland erbrachten Studien- und Prüfungsleistungen in deutlich kürzerer als der Regelstudienzeit erwerben lässt. An die Stelle des nicht existierenden Verfahrens zur Anerkennung des ausländischen Abschlusses tritt gewissermaßen die hochschulinterne Teil-Anerkennung von Studien- und Prüfungsleistungen vor dem Hintergrund der jeweiligen Studien- und Prüfungsordnung.

Aus dieser Überlegung resultierte das Pilotprojekt ProSALAMANDER (Programm zur Stärkung ausländischer Akademiker durch Nachqualifizierung an den Universitäten Duisburg-Essen und Regensburg): In unterschiedlichen regionalen Kontexten und bei teilweise gleichen, teilweise jedoch auch bewusst unterschiedlichen wählbaren Studiengängen wird erprobt, wie sich derartige Anrechnungsprozesse optimiert gestalten lassen und welche Formen der Nachqualifizierung sich als besonders wirksam erweisen. Von 2012 bis 2015 konnten durch die finanzielle Unterstützung seitens der Stiftung Mercator zwei Pilot-Jahrgänge mit insgesamt 64 Stipendien gefördert werden. Eine finanzielle

6 Für diese Fälle und auf Grundlage der Lissabon-Konven tion (aber nicht beschränkt auf Herkunftsländer, die diese ratifiziert haben) gibt es die Zentralstelle für ausländisches Bildungswesen (ZAB), die gegen eine Gebühr von $200 €$ eine Zeugnisbewertung durchführt. „Die Zeugnisbewertung soll den Zugang zum deutschen Arbeitsmarkt erleichtern. Sie ist ein offizielles Dokument, mit dem die ausländische Hochschulqualifikation beschrieben und ihre beruflichen und akademischen Verwendungsmöglichkeiten bescheinigt werden. Mit der Zeugnisbewertung wird eine vergleichende Einstufung vorgenommen: Die Zeugnisbewertung nennt den deutschen Bildungsabschluss, mit dem der ausländische vergleichbar ist, und informiert über die Möglichkeiten der Fortsetzung des Studiums sowie die Verfahren zur beruflichen Anerkennung. Das Verfahren der Zeugnisbewertung ist nicht mit einer beruflichen Anerkennung gleichzusetzen.,', http://www.anerkennungin-deutschland.de/html/de/zeugnisbewertung_von_hochschulabschluessen.php (letzter Zugriff 16.05.2015).

7 Die Erfahrung zeigt, dass gerade in den ehemaligen GUSStaaten von einer stringenten Einführung gestufter Studiengänge erst seit Kurzem die Rede sein kann. 
Existenzsicherung ist bei der vorliegenden Zielgruppe von besonderer Bedeutung, handelt es sich doch um Menschen mit bereits abgeschlossenem Studium und vielfach auch längerer Berufstätigkeit im Heimatland, die folglich zum einen älter sind als die durchschnittlichen inländischen Studierenden, zum anderen oft bereits familiäre Verpflichtungen haben, dabei jedoch nicht von den gängigen Studienförderungssystemen erfasst werden (siehe letzter Abschnitt).

Die Nachqualifizierung selbst ruht auf drei Säulen: Erstens den fachwissenschaftlichen Anteilen, für die keine Anrechnung auf den jeweiligen Studiengang vollzogen werden konnte und die demzufolge noch zu studieren sind. Eine Clearingstelle wirkt daran mit, dass diese Studienprogramme (bzw. die Anrechnungsverfahren) passgenau und individualisiert erstellt werden können. Zur Unterstützung der Teilnehmenden wurde darüber hinaus auch eine gezielte fachliche Beratung etabliert. Zweitens werden durch spezifisch erstellte Kursangebote (fach-)sprachliche Kenntnisse - in erster Linie in Deutsch, aber auch in Englisch - auf wissenschaftlichem und berufsfeldrelevantem Niveau vermittelt. Drittens trägt zur Erhöhung der Anstellungsfähigkeit wie zum Erfolg im deutschen Hochschulsystem auch die Förderung von methodischen Kompetenzen bei. Spezifische Angebote dieses Bereichs umfassen Trainings zur Erweiterung von interkulturellen kommunikativen Kompetenzen in Studium und Beruf oder zur Vorbereitung auf Bewerbungsgespräche, aber beispielsweise auch Kurse zum wissenschaftlichen Arbeiten und zur Gestaltung des Selbststudiums.

Das Spektrum der in diesem Programm wählbaren Fächer beinhaltete Studiengänge der Wirtschafts- und Ingenieurwissenschaften an der Universität Duisburg-Essen sowie Wirtschaftswissenschaften, Informations- und Medienwissenschaften, Medieninformatik sowie Sprach- und Kulturwissenschaften (kurz: IMSK) an der Universität Regensburg. Es handelte sich im betrachteten Zeitraum um eine Modellförderung mit Pilotcharakter, sodass die Anzahl der Teilnehmenden - schon aufgrund des finanziellen Rahmens der Stipendienvergabe - auf 32 Personen pro Jahrgang (dabei 16 je Universität), insgesamt also 64 Personen über die gesamte Projektlaufzeit, begrenzt wurde. In einem mehrstufigen Auswahlverfahren wurden - neben allgemeinen formalen Bedingungen - vor allem drei Kriterien angelegt: Umfang und Qualität von anrechenbaren Leistungen aus dem Studium im Heimatland, vorhandene Sprachkenntnisse sowie Darlegung der Motivation zur Teilnahme. Mittlerweile konnten erste Absolventen und Absolventinnen mit einem deutschen Bachelor- oder Masterabschluss verabschiedet werden. Aus den im Projekt gemachten Erfahrungen sowie den Ergebnissen der parallel durchgeführten, unabhängigen Evaluationen des Umfragezentrums Bonn ${ }^{8}$ lassen sich somit Aussagen zur Zusammensetzung der Teilnehmendenschaft sowie zu den hier ausgeprägten Mustern von typischerweise auftretenden Problemen bzw. Bedürfnislagen treffen.

\section{Die Teilnehmenden}

Zunächst ist auf den überdurchschnittlichen Anteil von Frauen bereits unter den Bewerbern, umso mehr aber unter den im Auswahlverfahren erfolgreichen Programmteilnehmenden einzugehen: Die Frauenquote lag bei etwa $60 \%$ unter den Bewerbungen (fächertypisch niedriger in den Ingenieurswissenschaften und höher in den kulturwissenschaftlich ausgerichteten Studiengängen). Unter den Teilnehmenden schließlich lag diese Quote sogar bei ca. $80 \%$. Dieser hohe Anteil erfolgreicher Bewerberinnen dürfte nicht zuletzt Ausdruck der Tatsache sein, dass Frauen der betreffenden Zielgruppe bei gleicher Qualifikation auf größere Schwierigkeiten hinsichtlich ihrer beruflichen Integration stoßen (s. o.). An dieser Stelle sind ungenutzte Potenziale für den hochqualifizierten Arbeitsmarkt zu vermuten, auch wenn sich nicht abschließend beurteilen lässt, in welchem Ausmaß diese unterproportionale Erwerbsbeteiligung auch traditionellen Karrierehemmnissen wie familiären Verpflichtungen geschuldet ist.

Ebenso aufschlussreich sind auch demografische Merkmale wie die regionale Herkunft und Altersstruktur der Programmteilnehmenden. Das erstgenannte Merkmal betreffend ließ sich eine große internationale Streubreite von Bewerbern wie Programmteilnehmenden ausmachen: Unter den Bewerbungen waren alle Kontinente mit Ausnahme von Nordamerika repräsentiert; allein in Duisburg-Essen wurden schließlich Personen aus 13 Nationen aufgenommen (unter denjenigen, die mittlerweile die deutsche Staatsangehörigkeit angenommen hatten, fanden sich noch sechs weitere Herkunftsländer). Dennoch lassen sich bestimmte Schwerpunktregionen von Herkunftsländern ausmachen: Die größte Gruppe unter den Bewerberinnen und Bewerbern wie unter den Teilnehmenden stammte aus ehemaligen GUS-Staaten (dabei lag jeweils Russland vor der Ukraine). Besonders niedrige Aufnahmequoten waren für Programminteressierte aus sub-saharischen afrikanischen Ländern sowie aus dem Nahen Osten zu verzeichnen, was große Unterschiede in den betreffenden nationalen Studienprogrammen und somit deutlich reduzierte Anrechnungsmöglichkeiten von Studienleistungen widerspiegelt. Von der Altersstruktur der aufgenommenen Kandidaten und Kandidatinnen her ließ sich mit der Gruppe der 26- bis 35-Jährigen ein Schwerpunkt identifizieren, wobei das Altersspektrum der Bewerber insgesamt von 24 bis 54 Jahre reichte.

8 Engin, T./Krüger, Th. (2013): Programmbegleitende Evaluation des Verbundprojekts ProSALAMANDER an den Universitäten Duisburg-Essen und Regensburg, gefördert durch die Stiftung Mercator GmbH, Zwischenbericht: uzbonn, Bonn; Engin, T./Krüger, Th. (2014): Programmbegleitende Evaluation des Verbundprojekts ProSALAMANDER an den Universitäten Duisburg-Essen und Regensburg, gefördert durch die Stiftung Mercator $\mathrm{GmbH}$, Fokusgruppen: uzbonn, Bonn. 
Aus den vorliegenden Biografien ließe sich so etwas wie eine typisierte Programmteilnehmende ableiten: ${ }^{9}$ Sie ist weiblich, Mitte Dreißig und kommt aus Russland. Dort hat sie einen oder mehrere Abschlüsse erworben und bereits mehrjährige Berufserfahrung in ihrem jeweiligen Fachgebiet gesammelt. Sie ist verheiratet und zog aus familiären Gründen nach Deutschland. Dort fand sie keinen beruflichen Anschluss, obwohl sie Deutschkurse belegte und Weiterbildungen absolvierte. Um sich zu betätigen und Geld zu verdienen, nahm sie eine nicht ausbildungsadäquate Beschäftigung an. Um wieder Zugang zum akademischen Arbeitsmarkt zu erlangen und in ihrem Fach auf den aktuellen Stand gebracht zu werden, bewarb sie sich bei ProSALAMANDER und studiert nun in einem ihrem Erststudium verwandten Studiengang. Laut den Umfrageergebnissen der letzten durchgeführten Evaluation beschäftigen sie zwei Fragenkomplexe besonders: Zum einen der Abgleich von Erwartungen an das Studium mit der Realität (Mehrfachbelastung durch familiäre oder berufliche Verpflichtungen; Unsicherheit bezüglich des späteren Arbeitsmarkterfolges); zum anderen die Frage der Finanzierung der Studienphase - dies insbesondere auch, nachdem die Stipendienunterstützung mit Frühjahr 2015 ausläuft: 2014 nahm in Duisburg-Essen die erste Kohorte von Programmteilnehmenden ohne derartige finanzielle Unterstützung das Studium auf. Die finanziellen Sorgen können sich bis zur Erwägung eines Studienabbruchs steigern, denn der Teilnehmenden stehen keine alternativen Finanzquellen offen und neben Studium und ggf. familiären Verpflichtungen kann sie kaum einer zusätzlichen Erwerbstätigkeit nachgehen.

Der Verzicht auf den Studienabschluss wäre jedoch nicht nur für die betroffenen Personen, sondern auch volkswirtschaftlich ein Verlust, wie eine im Rahmen des Projekts beim Institut der deutschen Wirtschaft Köln in Auftrag gegebene Studie belegt. Untersucht wurden hier zunächst die Wertschöpfungsgewinne, die Bildungsausländer durch akademische Nachqualifizierung erzielen können (wobei unter Wertschöpfung Bruttolöhne und Fiskalbeiträge verstanden wurden). Die durchschnittliche jährliche Wertschöpfung von Personen mit Studienabschluss im Ausland liegt dabei deutlich unter derjenigen, die von Personen mit ausländischem Schul- und inländischem Studienabschluss erzielt wurde: Im Zeitraum von ein bis fünf Jahren nach Einwanderung bzw. Beginn des Programms zur Nachqualifizierung erzielt die erstgenannte Gruppe demzufolge im Durchschnitt eine Wertschöpfung von $34.095 €$ pro Jahr, die zweite von $48.530 € .^{10}$ (Dass die entsprechenden Werte von Personen mit inländischem Schul- und Studienabschluss mit 53.277 € noch einmal deutlich darüber liegen, sei nur am Rande vermerkt.) Diese Marge wird mit zunehmender Zeit nach Einwanderung und somit Integration in den Arbeitsmarkt zwar etwas relativiert, bleibt aber als grundsätzlich positiver Effekt der Nachqualifizierung stets vorhanden, sodass die akkumulierten Gewinne bei einem Zeithorizont von 20 Jahren auf über $100.000 €$ anstiegen. ${ }^{11}$ Natürlich ist dabei auch zu berücksichtigen, dass die an einem Nachqualifizierungsprogramm partizipieren- den Personen während der Dauer ihrer Teilnahme - angesetzt wurden drei Semester - dem Arbeitsmarkt nicht zur Verfügung stehen und somit keine Wertschöpfung erzielen. Doch rechnet sich diese Investition für sie bereits nach einem überschaubaren Zeitraum von ca. fünf Jahren im Sinne eines Wertschöpfungsgewinns diskontierter Gesamtwerte. ${ }^{12} \mathrm{Ge}-$ samtfiskalisch rechnet sich die Ausrichtung eines derartigen Programms nach einem etwas längeren Zeitraum: Nach Abzug der Programmkosten ergäbe sich nach etwa 15 Jahren die saldierte Gewinnschwelle, selbst wenn man die Teilnehmenden mit einem rückzahlungsfreien Stipendium ausstattet, was die Förderung von Personen mit entsprechend langem zukünftigen Erwerbsleben nahelegen würde. ${ }^{13}$

\section{Probleme der Unterhaltssicherung}

Nach dem Auslaufen der Stipendienförderung durch die Stiftung Mercator kämen zur Unterhaltssicherung während der Nachqualifizierung nur noch Leistungen nach dem Bundesausbildungsförderungsgesetz (BAFöG) infrage. Entsprechende Anträge werden aber häufig abgelehnt, weil die Betroffenen die Altersgrenze überschritten haben und nicht glaubhaft machen können, dass sie seit ihrer Ankunft in Deutschland ein erneutes Studium angestrebt und lediglich wegen äußerer Gründe wie z. B. Kindererziehung daran gehindert waren (was Ausnahmen von der Altersgrenze begründen könnte). Außerdem darf es sich bei der Nachqualifizierung weder um ein Zweitstudium noch um einen Fachwechsel handeln; für die Verwertbarkeit auf dem deutschen Arbeitsmarkt kann es aber in vielen Fällen vorteilhaft sein, auf ein dem ursprünglichen Studienfach verwandtes Fach umzusteigen (z. B. von Psychologie auf Sozialpädagogik).

Viele an der akademischen Nachqualifizierung Interessierte befinden sich als Langzeit-Erwerbslose oder Alleinerziehende im Leistungsbezug nach dem SGB II. Wer im Leistungsbezug nach dem SGB III oder SGB II ein Anerkennungsverfahren nach dem BQFG mit dem Ergebnis durchläuft, dass vor einer Anerkennung ,wesentliche Unterschiede" durch Weiterbildungsmaßnahmen ausgeglichen werden müssen, kann während der Teilnahme an solchen Maßnahmen die betreffende Leistung weiter beziehen. ${ }^{14}$ Bei

9 Die Biografien derTeilnehmenden an der Universität Duisburg-Essen lassen sich nachlesen unter: https://www. uni-due.de/prosalamander/prosalamander.shtml.

10 Geis et al., a.a.O., S. $29 f$.

11 Ebd., S. 32.

12 Ebd., S. 33

13 Ebd., S. 39ff.

14 Bundesministerium für Bildung und Forschung (2014): Bericht zum Anerkennungsgesetz, S. 130, http://dipbt.bundestag.de/dip21/btd/18/010/1801000.pdf. 
Teilnehmenden einer akademischen Nachqualifizierung mit dem Ziel eines deutschen Hochschulabschlusses gilt dieses jedoch nicht, weil der überkommenen Grundauffassung der Arbeitsförderung zufolge „Arbeitnehmereigenschaft“ und „Studierendeneigenschaft" sich gegenseitig ausschließen und weil das Studium „dem Grunde nach“ durch Leistungen nach dem BAföG gefördert werden könnte. Dass diese Förderung in den meisten Fällen nicht gewährt wird, ändert nichts an der grundsätzlichen Nachrangigkeit von Leistungen nach dem SGB II gegenüber dem BAföG ( $\$ 7$ Abs. 5 SGB II). Paradoxer Weise könnten jedoch Leistungen weiter bezogen werden, wenn es sich um ein Studium in Teilzeit oder um ein Promotionsstudium handeln würde, weil hier das BAföG grundsätzlich ausscheidet. Jedoch werden die fraglichen Studiengänge nicht in Teilzeit angeboten, und eine doppelt so lange Studiendauer wäre für die Betroffenen auch kaum durchzuhalten.

Im Ergebnis ist festzuhalten, dass die von Einwanderern zunehmend nach Deutschland mitgebrachten, im Ausland erworbenen akademischen Qualifikationen auf dem deutschen Arbeitsmarkt oft nicht adäquat verwertet werden können und dass für einen erheblichen Teil der Fälle weder ein Anerkennungsverfahren noch eine Unterhaltsförderung beim Erwerb eines deutschen Abschlusses existieren. Dadurch wird in einer Situation von Fachkräfteengpässen Humankapital entwertet, und vorhandene Qualifizierungspotenziale von hoch motivierten Menschen bleiben ungenutzt. Durch ein auf die Situation der Zielgruppe zugeschnittenes Stipendienprogramm - sei es als Sonderbestimmung im BAföG, sei es als eigenständiges Programm im Rahmen der Integrationsförderung - ließe sich dieses Problem lösen bei insgesamt überschaubaren Zahlen von Interessierten und folglich von Kosten.

\section{AUTOREN}

ANNA KATHARINA JACOB, Dr., Universität Duisburg-Essen, arbeitet am Zentrum für Hochschul- und Qualitätsentwicklung und leitet dort den Projektbereich Offene Hochschule. Arbeitsschwerpunkte: Internationale Hochschulforschung und Diversity Management.

katharina.jacob@uni-due.de

MATTHIAS KNUTH, Prof. Dr., ist Soziologe und forscht am Institut Arbeit und Qualifikation (IAQ) der Universität DuisburgEssen. Arbeitsschwerpunkte: Arbeitsmarktpolitik, Alterserwerbstätigkeit und Arbeitsmigration.

matthias.knuth@uni-due.de 